\title{
Survival analysis of genome-wide profiles coupled with Connectivity Map database mining to identify potential therapeutic targets for cholangiocarcinoma
}

\author{
PENG LIN ${ }^{1}$, XIAO-ZHU ZHONG ${ }^{1}$, XIAO-DONG WANG ${ }^{1}$, JIAN-JUN LI ${ }^{2}$, RUI-QI ZHAO ${ }^{3}$, YU HE $^{3}$, \\ YAN-QIU JIANG ${ }^{3}$, XIAN-WEN HUANG ${ }^{4}$, GANG CHEN $^{3}$, YUN HE ${ }^{1}$ and HONG YANG ${ }^{1}$ \\ ${ }^{1}$ Department of Medical Ultrasonics, First Affiliated Hospital of Guangxi Medical University; \\ ${ }^{2}$ Department of General Surgery, The Second Affiliated Hospital of Guangxi Medical University; \\ Departments of ${ }^{3}$ Pathology and ${ }^{4}$ Traditional Chinese Medicine, First Affiliated Hospital of Guangxi Medical University, \\ Nanning, Guangxi Zhuang Autonomous Region 530021, P.R. China
}

Received May 6, 2018; Accepted September 3, 2018

DOI: $10.3892 /$ or.2018.6710

\begin{abstract}
Cholangiocarcinoma (CCA) is one of the most common epithelial cell malignancies worldwide. However, its prognosis is poor. The aim of the present study was to examine the prognostic landscape and potential therapeutic targets for CCA. RNA sequencing data and clinical information were downloaded from The Cancer Genome Atlas (TCGA) dataset and processed. A total of 172 genes that were significantly associated with overall survival of patients with CCA were identified using the univariate Cox regression method. Bioinformatics tools were applied using the Kyoto Encyclopedia of Genes and Genomes (KEGG) and gene ontology (GO). It was identified that 'Wnt signaling pathway', 'cytoplasm' and 'AT DNA binding' were the three most significant GO categories of CCA survival-associated genes. 'Transcriptional misregulation in cancer' was the most significant pathway identified in the KEGG analysis. Using the Drug-Gene Interaction database, a drug-gene interaction network was constructed, and 31 identified genes were involved in it. The most meaningful potential therapeutic targets were selected via protein-protein and gene-drug interactions. Among these genes, polo-like kinase 1 (PLK1) was identified to be a potential target due to its significant upregulation in CCA. To rapidly find molecules that may affect these genes, the Connectivity Map was queried. A series of molecules were selected for their potential anti-CCA functions. 0297417 0002B and tribenoside exhibited the highest connection scores
\end{abstract}

Correspondence to: Dr Hong Yang, Department of Medical Ultrasonics, First Affiliated Hospital of Guangxi Medical University, 6 ShuangYong Road, Nanning, Guangxi Zhuang Autonomous Region 530021, P.R. China

E-mail: yanghong@gxmu.edu.cn

Key words: cholangiocarcinoma, connectivity map, molecular docking, prognosis, polo-like kinase 1 with PLK1 via molecular docking. These findings may offer novel insights into treatment and perspectives on the future innovative treatment of CCA.

\section{Introduction}

Cholangiocarcinoma (CCA) is an epithelial cell malignancy originating from cholangiocytes $(1,2)$. CCA, together with liver cancer, is reported to be the fifth leading cause of cancerassociated mortality in 2018 in the USA (3). Currently, surgical resection remains the mainstay of curative treatment, although it is only available for selected patients (4). Poor prognosis, even with ideal surgery, is the key problem in CCA. The 5-year overall survival (OS) rate of CCA is $15-40 \%$, and the postsurgical recurrence rate is $50-60 \%$ (5-8).

A number of other therapies have been used in CCA. Local advanced and metastatic CCA is treated with systemic chemotherapy; cisplatin and gemcitabine are the common drugs, and the drug yttrium 90 is used in local radiotherapy (9). Currently, there are few reports on drug treatments for CCA. Studies suggest that 14-deoxy-11, 12-didehydroandrographolide and curcumin may be the potential drugs for the treatment of CCA $(10,11)$. Even with the large number of tested drugs, the results remain unsatisfactory. The principal causes may be the lack of enrollment in trials and the limited number of biological profiles of CCA (12). Hence, novel and effective drugs are urgently required. Connectivity Map (CMap) is a database containing 7,000 gene expression data profiles treated by $\sim 1,300$ compounds (13). In the field of drug development, it is helpful to make rapid use of gene expression profiles associated with diseases. This approach may identify the principal chemical structure of the majority of drug molecules and elucidate the possible mechanism of drug action.

To examine novel therapeutics for CCA, the present study screened the survival-associated genes of CCA, which were identified based on gene expression profiles provided by The Cancer Genome Atlas (TCGA) database. Simultaneously, pathways associated with poor survival in CCA were ascertained 
through gene-set functional enrichment analysis. Using the associations already obtained, the present study put forward a model of the network aimed at these prognostic-related targets. Finally, through CMap, a number of molecules associated with the prognosis of CCA were screened out. In brief, the aim of the present study was to examine the prognostic landscape and potential therapeutic targets of CCA.

\section{Materials and methods}

Processing of genomic data from TCGA dataset. RNA sequencing data and clinical information from 45 patients with CCA were download from TCGA portal (https:// cancergenome.nih.gov/). The mRNA expression profiles were extracted, and the data were normalized in the form of transcripts per kilobase million (TPM). To obtain a more moderate result, mRNAs with an average TPM $<1$ were removed.

Survival analysis and functional annotation. The association between OS and the gene expression profiles was evaluated by univariate Cox regression analysis, which was conducted using the survival package in R software version 3.44 (https:// CRAN.R-project.org/package=survival). Subsequently, the prognostic genes were submitted to DAVID Bioinformatics Resources 6.8 (https://david.ncifcrf.gov/) for functional enrichment and pathway analyses, which included the Kyoto Encyclopedia of Genes and Genomes (KEGG) and gene ontology (GO). The functional enrichment results were displayed using the ggplot2 package in R software (https:// CRAN.R-project.org/package=ggplot2), and Cytoscape software version 3.61 (http://www.cytoscape.org/). Furthermore, the protein-protein interactions (PPIs) among these prognostic genes were generated. Interaction data were downloaded from the STRING online database (http://string-db.org/), and further visualization was performed using Cytoscape software.

Gene-drug interaction network. To study the potential survival-associated gene therapeutic targets based on the available resources, the present study used the online Drug-Gene Interaction database (DGIdb), which stores information about druggability and interactions between drugs and genes from the published literature, databases and web resources (14). Based on this information, the drug-gene interaction network was built to identify druggable targets.

CMap analysis. CMap provides perspectives on small-molecule drugs, gene expression, and diseases that are associated with each other at the genome level. Therefore, it allows researchers to link gene datasets with drugs that are closely associated with the disease process. The secondary use of drugs in another disease greatly reduces the time and expense required for drug development. To find novel drugs to alter the poor OS of patients with CCA, the survival-associated genes were uploaded to CMap, compared and ranked via the Kolmogorov-Smirnov test. A drug with a positive score indicates that there is a similar effect or mechanism of poor survival for the imported drug. Small molecules with negative scores were selected for further analysis, as this indicated that these molecules may work in the improvement of poor survival in patients with CCA.
Potential targets of cholangiocarcinoma. Potential therapeutic targets for improving the survival of patients were also identified. Hub genes of the PPI network were selected when the number of connections was $>5$. Genes that were significant in the development of drugs and possessed druggability were also identified in the drug-gene network. Genes obtained by the above two methods simultaneously were considered to be potential targets for CCA.

Molecular docking analysis. To verify the targeting association between the molecules and targets, the Sybyl X-2.0 Surflex-Dock program (Certara, Princeton, NJ, USA) was used to calculate the molecular docking affinity index. The program obtained detailed information about the combinations of novel drugs and targets, and the docking scores were calculated to represent the binding affinities.

\section{Results}

Prognostic evaluation of mRNAs in cholangiocarcinoma. Following omission of the genes with low abundance, 14,458 genes were obtained for prognostic evaluation. The univariate Cox regression method identified a total of 172 genes that were significantly associated with the OS of patients with CCA. Among these genes, 94 genes had a tumor suppressor role, and 78 were oncogenic genes. The top five most significant survival-associated genes are displayed in Fig. 1.

Biological processes of survival-associated genes. In the biological processes-associated subgroup of GO terms, the survival-associated genes were significantly enriched in 'Wnt signaling pathway', 'apoptotic process' and 'transcription from RNA polymerase I promoter' (Fig. 2A). In addition, the cell component analysis revealed that these genes were most enriched in 'cytoplasm', 'senescence-associated heterochromatin focus' and 'collagen type IV trimer' (Fig. 2B). 'AT DNA binding', 'signal transducer activity' and 'protein serine/ threonine kinase activity' were the three most significant categories for molecular function (Fig. 2C). Furthermore, the KEGG pathway analysis identified a number of oncogenic pathways, including 'transcriptional misregulation in cancer', which may be the altered pathways in patients with poorer survival (Fig. 3A). The PPI network revealed that ubiquitin $\mathrm{C}$ (UBC), Jun proto-oncogene, AP-1 transcription factor subunit (JUN) and polo-like kinase 1 (PLK1) ranked as core genes within the group of genes (Fig. 3B), which indicated that these genes may be the most important in the network.

Drug-gene network analysis. Based on the interactions between the drugs and the effects of unknown molecule drugs, the drug-gene interaction network was constructed. A total of 31 genes identified to be putative molecular targets for antitumor therapy were involved in the drug-gene network (Fig. 4). These drugs may have the potential to be effective anti-CCA drugs in the future.

Candidate drugs for CCA therapy. From the small molecules with a negative score that had potential against CCA, a total of 49 small molecule drugs were collected by using the CMAP database. The top ten significant novel drugs were LY-294002, 
A
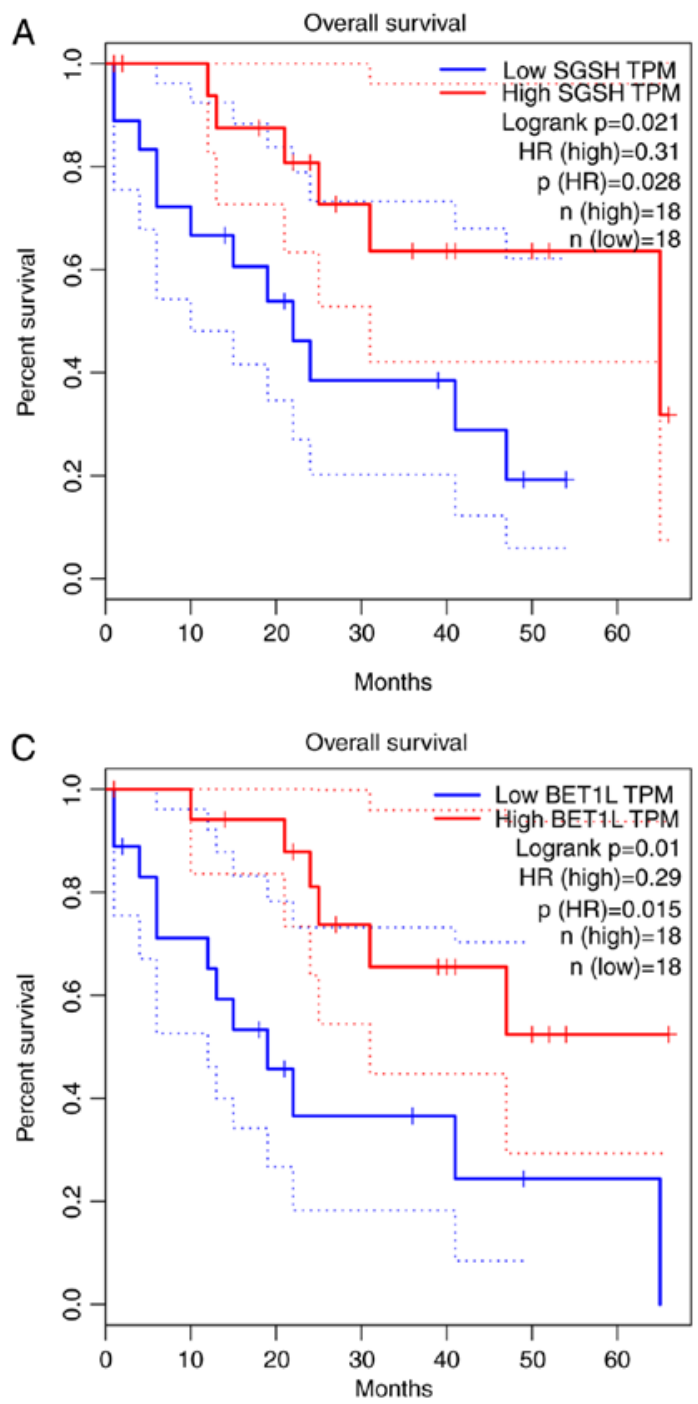

B

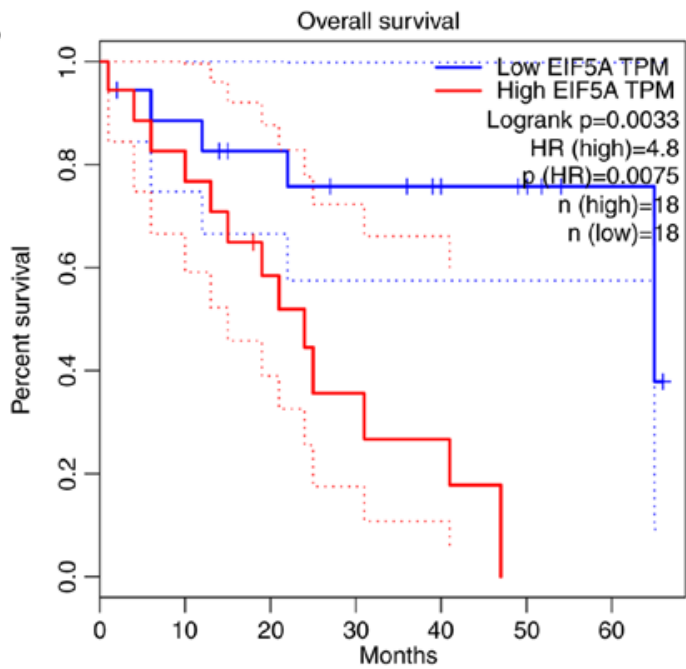

D

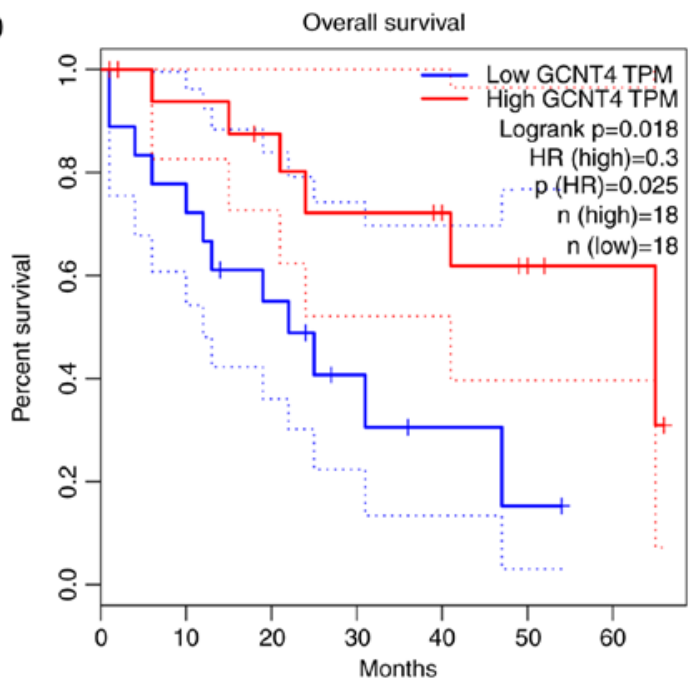

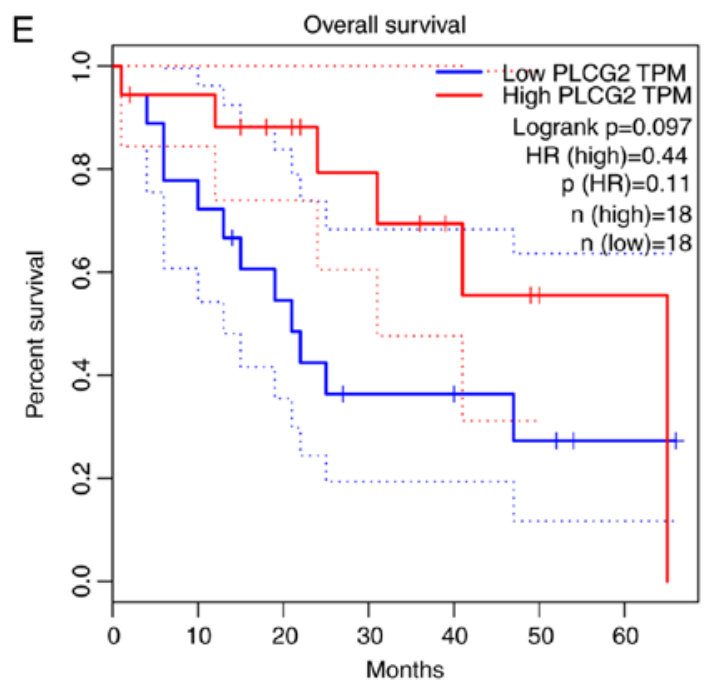

Figure 1. Top five most significant survival-associated genes in cholangiocarcinoma. (A) SGSH; (B) EIF5A; (C) BET1L; (D) GCNT4; (E) PLCG2. SGSH, N-sulfoglucosamine sulfohydrolase; EIF5A, eukaryotic translation initiation factor 5A; BET1L, Bet1 golgi vesicular membrane trafficking protein-like; GCNT4, glucosaminyl (N-acetyl) transferase 4, core 2; PLCG2, phospholipase C $\gamma 2$; HR, hazard ratio; TPM, Transcripts Per Kilobase Million.

trichostatin A, cephaeline, 0297417-0002B, quinostatin, puromycin, tribenoside, nabumetone, $15-\delta$ prostaglandin $\mathrm{J} 2$ and scoulerine (Fig. 5). The chemical structures of these drugs are displayed in Fig. 6.
Potential target selection. To select the most significant targets among the group of survival-associated genes, the PPI network was analyzed, and it was demonstrated that angiotensin II receptor type 1, arachidonate 5-lipoxygenase, 
A

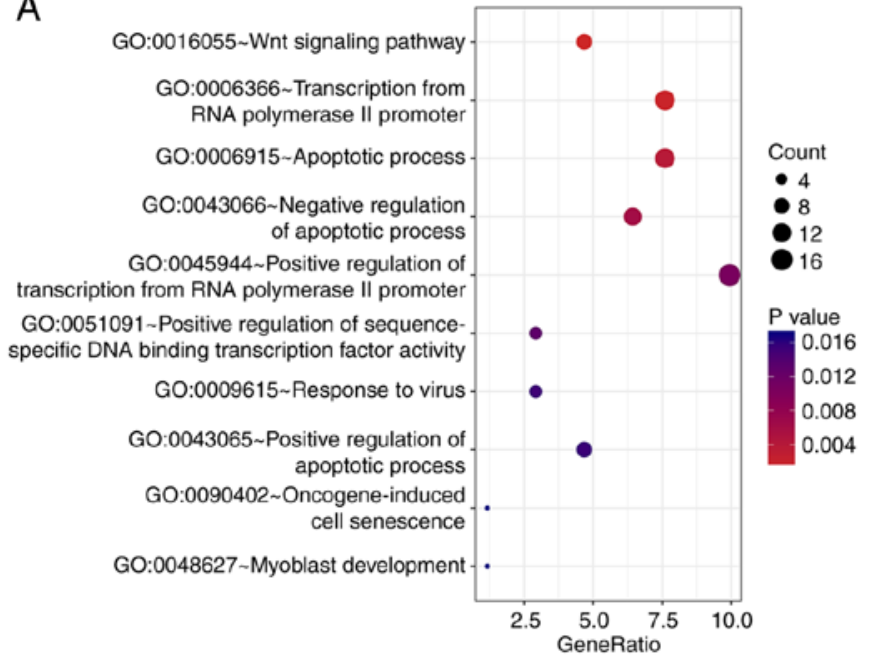

$\mathrm{B}$

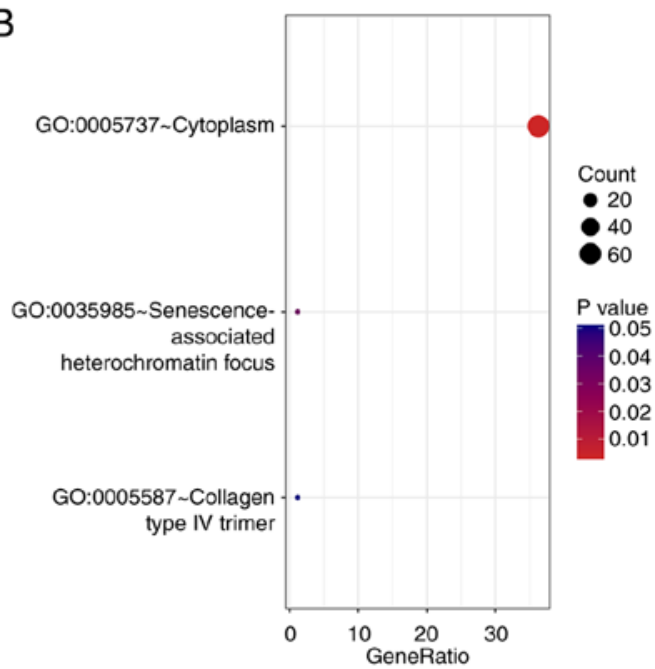

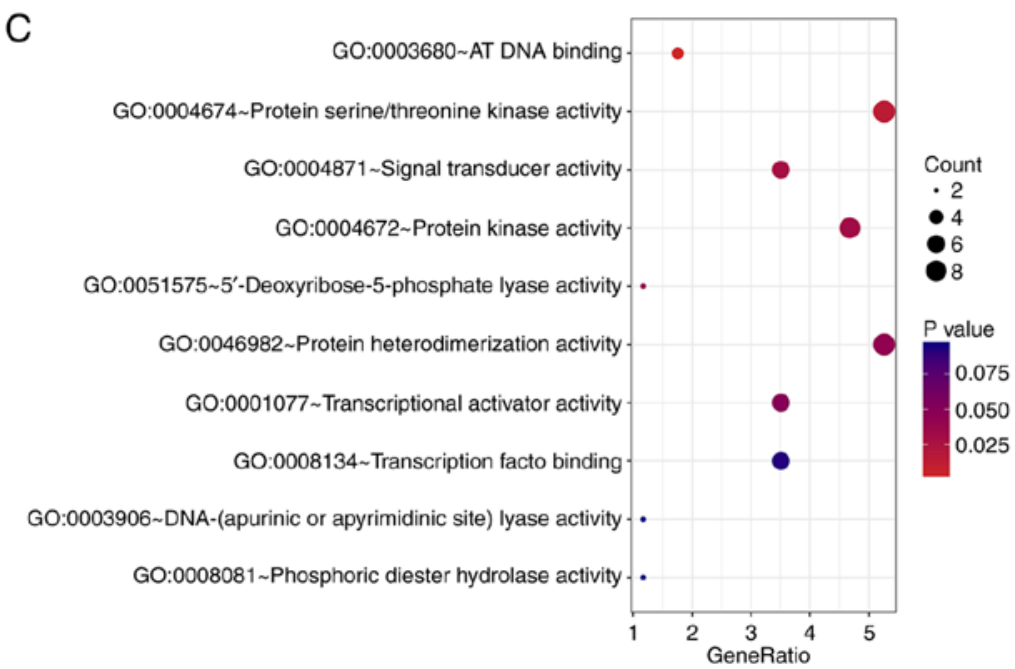

Figure 2. GO analysis of cholangiocarcinoma survival-associated genes. (A) Biological process; (B) cellular component; (C) molecular function. GO, gene ontology.

PLK1, $\alpha$-fetoprotein, lysine methyltransferase 2A, natriuretic peptide $\mathrm{C}$, TTK protein kinase and phosphatidylethanolamine N-methyltransferase were the hub genes (Fig. 7A). The druggene network analysis demonstrated that JUN, UBC, PLK1, KMT2A, interferon regulatory factor 7 and early growth response 1 had potential as drug targets (Fig. 7B). Molecules targeting these genes may have improved druggability. Notably, PLK1 was simultaneously ranked within the core genes of the two networks (Fig. 7C). PLK1 was significantly upregulated in CCA tumor samples compared with non-tumor samples (Fig. 7D).

Correlation between PLK1 and compounds via molecular docking. To examine whether the potential anti-CCA effects of these molecules were due to direct targeting between the compounds and genes, molecular docking analysis was performed. PLK1, one of the hub survival-associated genes, was selected. Notably, the top ten molecules (Fig. 8A) all exerted moderate binding capacity with PLK1. 02974170002B and tribenoside exhibited the highest connection scores (Fig. 8B and C). These findings may provide novel anti-CCA insights for the future.

\section{Discussion}

The present study indicated that certain genes may be identified as candidate molecular targets that may serve an essential role in the prognosis and treatment of CCA. The GO enrichment and KEGG pathway analysis results indicated that these target genes were closely associated with the relevant signaling pathways and processes of tumorigenesis, including 'transcription from RNA polymerase I promoter' and 'Wnt signaling pathway'. Networks associated with the prognosis of these target genes were constructed, demonstrating that the genes were potential targets of a number of drugs. Furthermore, the CMap search identified certain novel compounds with potential therapeutic value for CCA.

CCA is a bile duct malignancy, which is difficult to accurately diagnose and has a poor prognosis (15). Patients diagnosed with CCA are likely to progress into the advanced stages. In the absence of efficient therapeutic methods in the clinic, the morbidity and mortality rates of CCA have been increasing gradually in recent years (1). Therefore, the present study comprehensively analyzed CCA survival-associated genes, and key findings emerged 

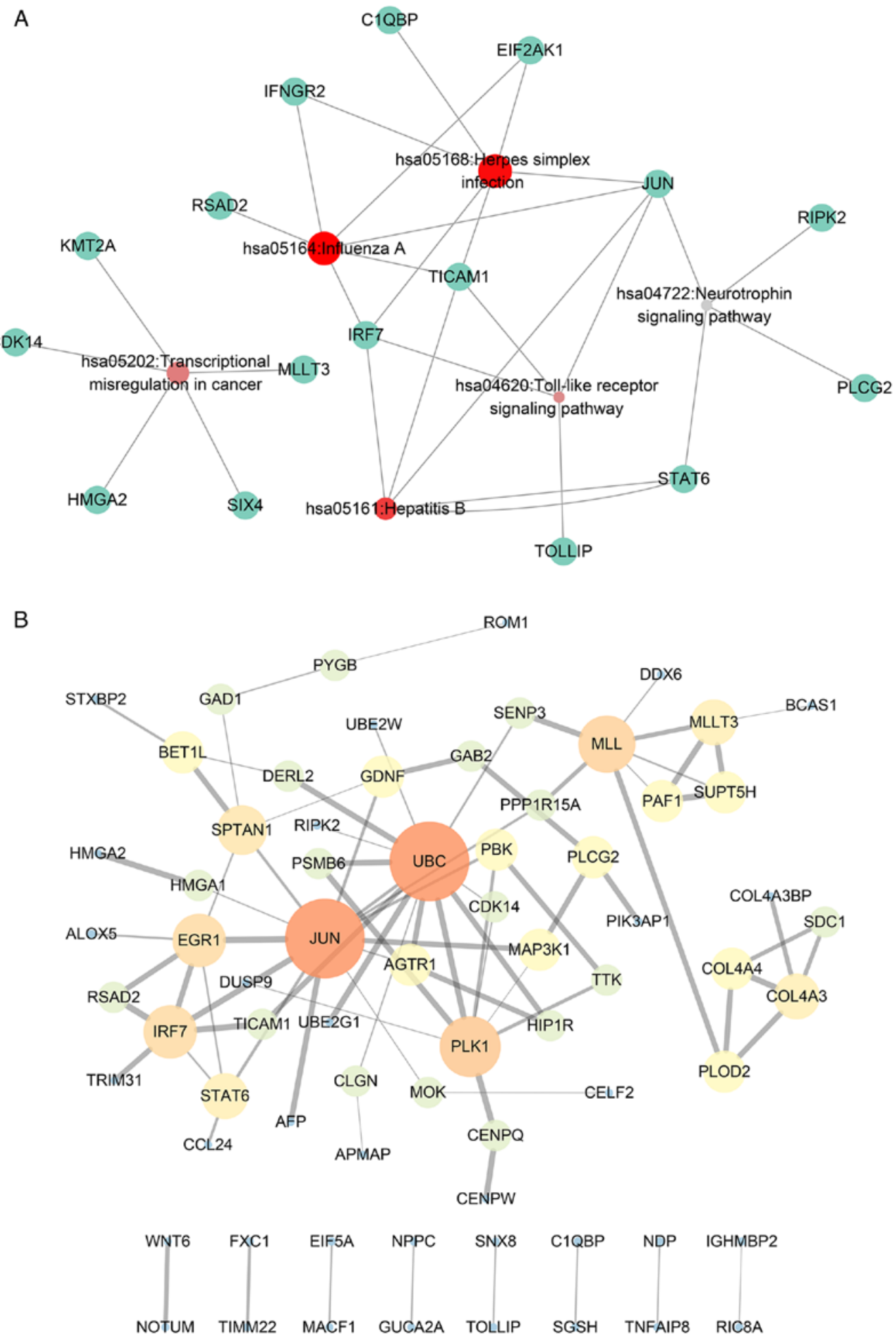

Figure 3. KEGG analysis and PPI network of cholangiocarcinoma survival-associated genes. (A) KEGG pathway analysis revealed significant pathways. Green represents survival-associated genes and red represents enriched pathways. (B) The PPI network revealed the interactions of genes at the protein level. KEGG, Kyoto Encyclopedia of Genes and Genomes; PPI, protein-protein interaction.

regarding target genes [N-sulfoglucosamine sulfohydrolase (SGSH), eukaryotic translation initiation factor 5A (EIF5A), Bet1 golgi vesicular membrane trafficking protein-like (BET1L), glucosaminyl (N-acetyl) transferase 4, core 2 (GCNT4) and phospholipase C $\gamma 2$ (PLCG2)] that may be associated with the prognosis of CCA. SGSH is one of a number of enzymes that are associated with the lysosomal degradation of heparan sulfate (16). EIF5A is associated with protein synthesis and cell proliferation. High eIF5A expression may be observed in various types of tumors and is responsible for cancer metastasis (17). Altered glycosylation is considered to be one of the most common cancer biomarkers (18). PLCG2 is able to provide survival signals for cells, inhibit cellular apoptosis, and indirectly activate protein kinase $\mathrm{C}$, which is frequently highly expressed in cancer tissues (19). 


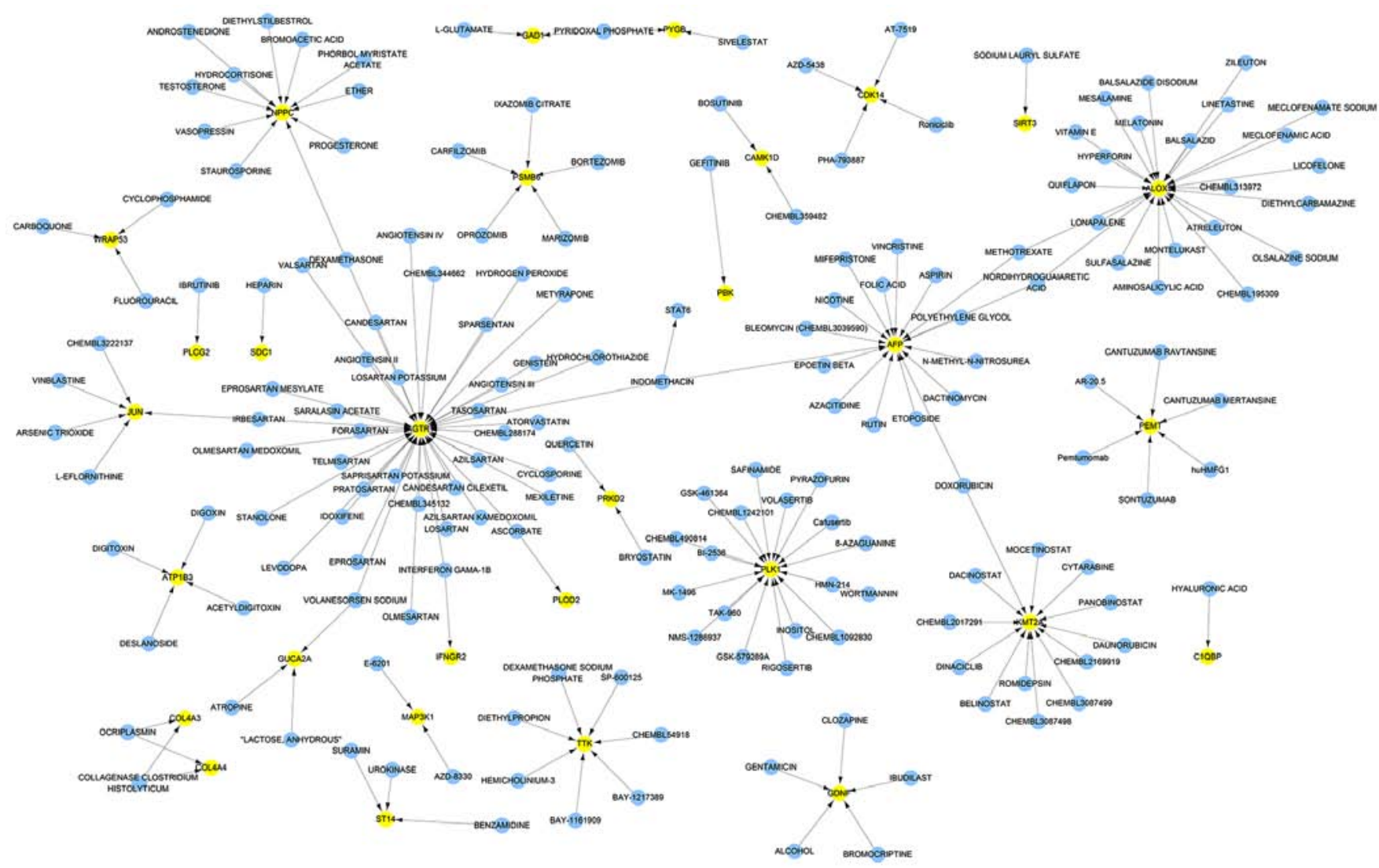

Figure 4. Drug-gene interaction network. Yellow represents significant survival-associated molecular targets and blue indicates drugs known to inhibit the targets.

\begin{tabular}{|c|c|c|c|c|c|c|}
\hline Rank & Cmap Name & Mean & $\mathrm{N}$ & Enrichment & P-value & specificity \\
\hline 2 & LY-294002 & -0.345 & 61 & -0.337 & 0 & 0.2577 \\
\hline 3 & trichostatin A & -0.32 & 182 & -0.311 & 0 & 0.5248 \\
\hline 5 & cephaeline & -0.749 & 5 & -0.861 & 0.00008 & 0.0602 \\
\hline 8 & 0297417-0002B & -0.674 & 3 & -0.932 & 0.0005 & 0.0328 \\
\hline 9 & quinostatin & -0.785 & 2 & -0.983 & 0.00064 & 0.0534 \\
\hline 10 & puromycin & -0.658 & 4 & -0.854 & 0.00084 & 0.0373 \\
\hline 15 & tribenoside & -0.558 & 4 & -0.815 & 0.00215 & 0 \\
\hline 18 & nabumetone & -0.559 & 4 & -0.782 & 0.00462 & 0.0063 \\
\hline 20 & 15-delta prostaglandin $\mathrm{J} 2$ & -0.381 & 15 & -0.414 & 0.00747 & 0.2256 \\
\hline 22 & scoulerine & -0.506 & 4 & -0.751 & 0.00768 & 0.0168 \\
\hline
\end{tabular}

Figure 5. CMap analysis for selecting novel drugs which possess potential anti-cholangiocarcinoma function. CMap, Connectivity Map.

GO classification and KEGG pathway enrichment analysis was used in the present investigation to identify survival-associated genes. Certain notable GO biological processes were identified, including 'Wnt signaling pathway' and 'transcription from RNA polymerase II promoter'. The Wnt signaling pathway is essential for the survival of stem cells and the maintenance of the regenerative ability of tissues (20). The basic role of the Wnt/ $\beta$-catenin pathway in development requires regulatory control at multiple levels, and a deficiency at any level may lead to tumor formation (21). Previous studies have demonstrated that the proliferation, invasion and epithelial-mesenchymal transition of CCA cells are associated with Wnt/ $\beta$-catenin signaling $(22,23)$. $\mathrm{Wnt} / \beta$-catenin signaling is progressively activated during cholangiocarcinogenesis, which indicates that it may serve an essential role in the occurrence and prognosis of CCA. Transcription via RNA polymerase I is a highly dynamic process that is tightly regulated at each step of the transcription cycle (24). RNA polymerase I speeds up the cell cycle by promoting the transcription of genes, thereby promoting cell growth and tumor formation.

KEGG pathway analysis demonstrated that the survivalassociated genes were principally involved in the influenza A, herpes simplex infection and hepatitis B signaling pathways. When the host is infected with a virus, p53 performs an inhibitor role in tumorigenesis by promoting the host immune response and protecting the host from cancer (25). The excessive activation of Ras signals also increases the degree of clearance of tumor cells following infection with herpes simplex virus (e.g. R3616) (26). Bioinformatics analysis was also performed via PPI network analyses. Based on node connectivity, genes were classified as hub genes in CCA. UBC, JUN, and PLK1 were the three hub genes that were essential nodes in the gene co-expression network. 
A<smiles>O=c1cc(N2CCOCC2)oc2c(-c3ccccc3)cccc12</smiles>

D<smiles>Cc1cc(=O)n(C2CCCC2)c2nc(Nc3ccc(N4CCNC(C)(C)C4)cc3)ncc12</smiles>

B<smiles>CC(C=CC(=O)NO)C(=O)c1ccc(N(C)C)cc1</smiles>

C<smiles>CCC1CN2CCc3cc(OC)c(OC)cc3C2CC1CC1NCCc2cc(O)c(OC)cc21</smiles>

$\mathrm{E}$<smiles>CCc1ccc(C(=O)c2cnc3cc4c(cc3c2N2CCC(C(N)=O)CC2)OCCO4)cc1</smiles>

F

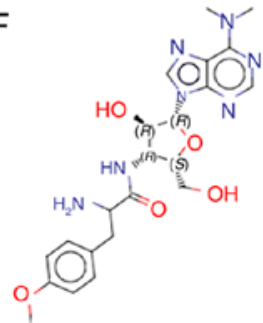

G<smiles>CCOC1O[C@@H](COCc2ccccc2)[C@H](OCc2ccccc2)[P@@]1O</smiles>

$\mathrm{H}$<smiles>COc1ccc2cc(CCC(C)=O)ccc2c1</smiles>

I<smiles>CCCCC/C=C\C[C@H]1C=C/C(=C\C=C/[13CH2])O1</smiles><smiles>COc1cc2c(cc1O)[Al]1Cc3ccc(OC)c(O)c3CN1CC2</smiles>

Figure 6. Chemical structures of the top ten molecules. (A) LY-294002; (B) trichostatin A; (C) cephaeline; (D) 0297417-0002B; (E) quinostatin; (F) puromycin; (G) tribenoside; (H) nabumetone; (I) 15- $\delta$ prostaglandin J2; (J) scoulerine.
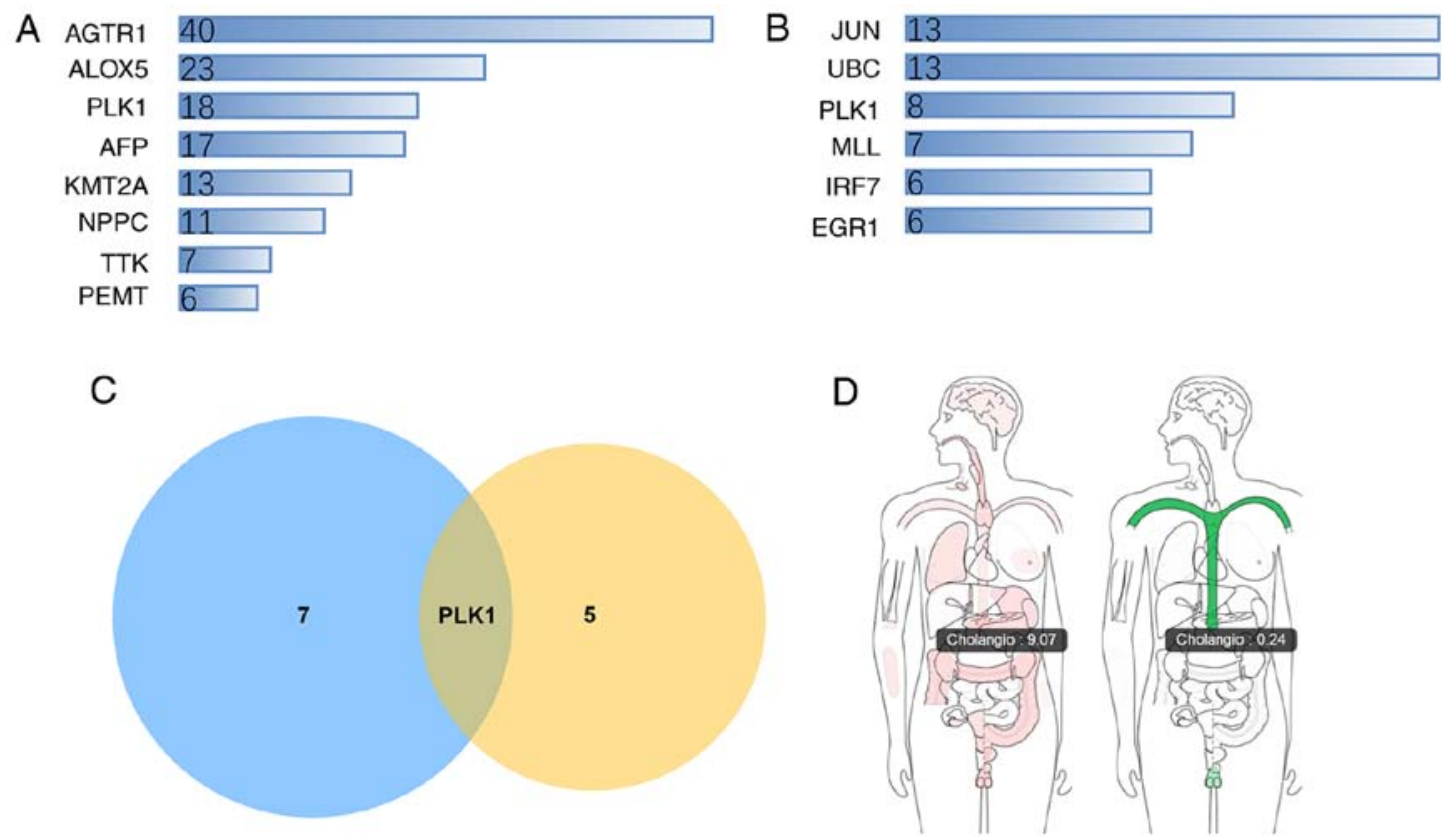

Figure 7. Selection of molecular targets of cholangiocarcinoma. (A) Hub genes in the drug-gene interaction network. (B) Hub genes in the protein-protein interaction network; (C) The intersection of the two networks. (D) The expression levels of PLK1 in cholangiocarcinoma and adjacent non-tumor tissues. PLK1, polo-like kinase 1. 
A

\begin{tabular}{|ccccc|}
\hline Molecules & Total score & Crash & Polar & Similarity \\
\hline LY-294002 & 3.9698 & -0.7693 & 0.0000 & 0.334 \\
\hline trichostatin A & 5.8162 & -1.2559 & 1.0574 & 0.258 \\
\hline cephaeline & 5.6541 & -1.0579 & 0.3888 & 0.357 \\
\hline 0297417-0002B & 6.2653 & -1.6817 & 0.2848 & 0.384 \\
\hline quinostatin & 5.1788 & -2.3197 & 1.0437 & 0.28 \\
\hline puromycin & 3.369 & -1.4079 & 0.8062 & 0.355 \\
\hline tribenoside & 6.9053 & -1.8815 & 0.0000 & 0.357 \\
\hline nabumetone & 3.6566 & -0.2961 & 1.1204 & 0.34 \\
\hline 15-delta prostaglandin J2 & 5.7681 & -2.7282 & 0.0000 & 0.325 \\
\hline scoulerine & 4.2851 & -0.8934 & 2.4170 & 0.258 \\
\hline
\end{tabular}

B

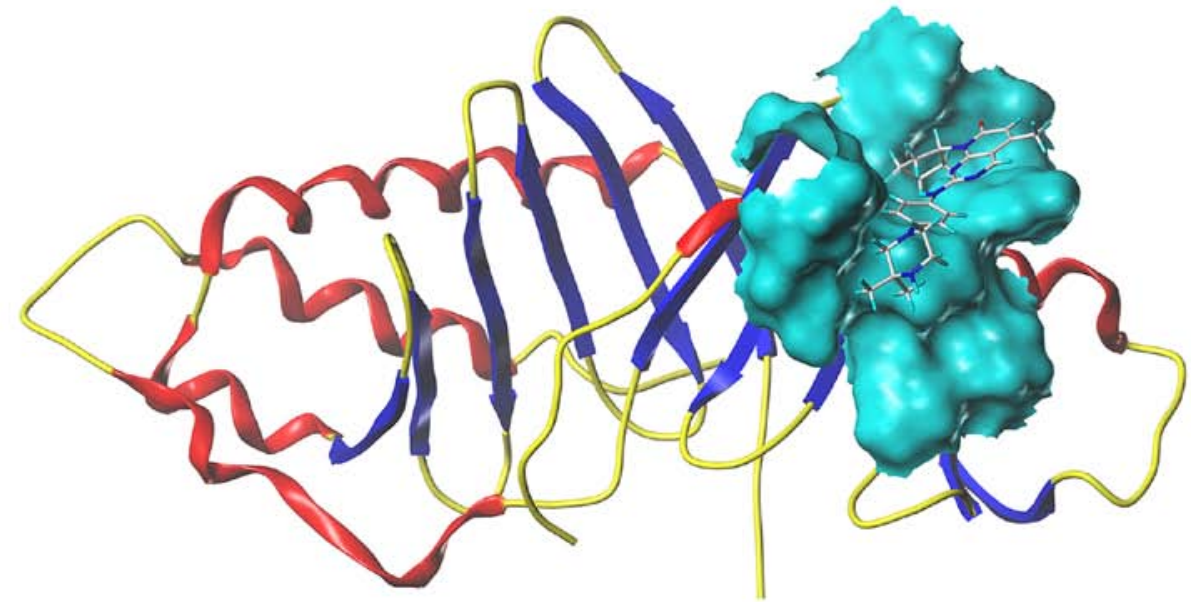

C

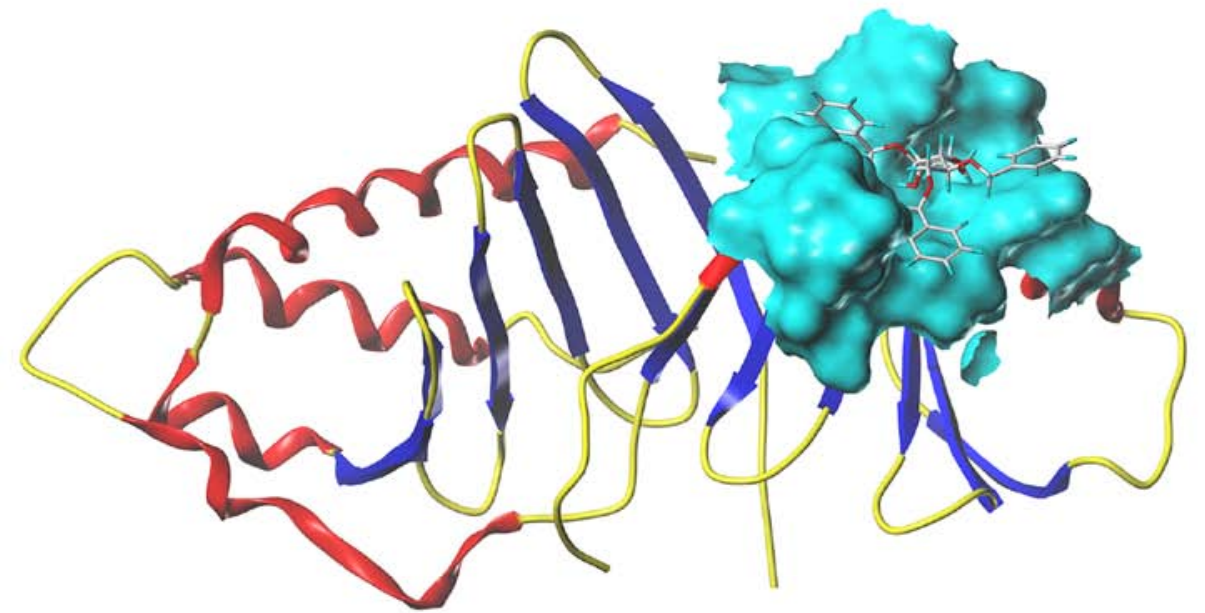

Figure 8. Molecular interaction analysis of predicted potential novel drugs docked to PLK1. (A) Binding affinity of these experiments. (B) Binding of 02974170002B and PLK1. (C) Binding of tribenoside and PLK1. PLK1, polo-like kinase 1.

UBC is also known as HMG20 and code for the ubiquitin gene. Ubiquitination has a crucial role in protein degradation, DNA repair, cell cycle regulation, kinase modification, endocytosis and the regulation of other cell signaling pathways (27). PLK1 is also termed STPK13 and encodes a serine/threonine protein kinase that is part of the CDC5/Polo subfamily. Studies have demonstrated that PLK1 is overexpressed in various types of tumors, and it is during the period of mitosis that PLK1 is observed to be highly expressed (28-30). With the decrease in this protein in carcinoma cells, PLK1 markedly suppresses cell proliferation and triggers cellular apoptosis; thus, PLK1 is considered to be a target for the treatment of carcinoma (31). JUN is regarded as the transforming gene associated with avian sarcoma virus 17 . The protein encoded by JUN has a high similarity with the viral protein, and the direct interaction between the protein encoded by JUN and the particular target DNA sequences may regulate gene expression. In addition to intron-less genes, JUN is mapped to 1p32-p31, a chromosomal region involved in translocations and deletions in human malignancies (32). The antitumor medicine volasertib is an effective selective PLK1 suppressor, which may cause selective G2/M blockade and apoptosis in various types of cancer cells $(33,34)$. Simultaneously, it induces reversible cell arrest at the G1 and G2 phase while protecting healthy cells from apoptosis. Volasertib has been used in trials studying the treatment of acute myeloid leukemia, neoplasms and myelodysplastic syndrome and may also serve a role in the treatment of CCA (35-37).

Through CMap analysis, 10 drugs (LY-294002, trichostatin A, Cephaeline, 0297417-0002B, quinostatin, puromycin, 
tribenoside, nabumetone, $15-\delta$ prostaglandin $\mathrm{J} 2$, and scoulerine) were identified that may have potential novel efficacy against the poor prognosis of CCA. Trichostatin A induces hyperacetylation of the core histone followed by structural modulation of chromatin, and finally causes the promotion of selective gene transcription and the suppression of tumor growth by reversibly inhibiting deacetylases. Currently, trichostatin A has been used in the treatment of laryngeal cancer and as a novel biomarker for nasopharyngeal carcinoma, and is also used to prevent the side effects of cisplatin in the treatment of cancer (38). It may have positive effects on CCA treatment. LY-294002 is a specific inhibitor of phosphatidylinositol 3-kinase (PI3K), which serves a role in the treatment of cancer by affecting the PI3K/RAC- $\alpha$ serine/threonine-protein kinase signaling pathway (39). Tribenoside and nabumetone are anti-inflammatory drugs, which may be used to inhibit the synthesis of prostaglandins and thrombin precursors to antiinflammatory, antipyretic and analgesic effect. Among them, nabumetone has been used to treat acute and chronic osteoarthritis and rheumatoid arthritis. Tribenoside has been used in the clinical treatment of hemorrhoidal disease associated with inflammation, coagulation, varicose veins and wounds $(40,41)$. Puromycin is a conversion inhibitor in biosynthesis and inhibits protein synthesis, and has anti-tumor, anti-parasitic and antibiotic effects (42). Cephaeline and scoulerine are associated with alkaloid synthesis (43). L-scoulerine antagonizes the dopamine D2 receptor to inhibit decreases in prepulse inhibition, which is caused by MK-801. It may be a potential cure for schizophrenia (44). 15- $\delta$ prostaglandin J2, 0297417-0002B and quinostatin have no clinical applications at present. $15-\delta$ prostaglandin $\mathrm{J} 2$ is a nuclear factor- $\mathrm{\kappa b}$ inhibitor (45). The above drugs may affect CCA in a variety of small molecular pathways.

It was further proposed that PLK1 may be a potential therapeutic target at the genome level. Previously, Thrum et al (46) reported that the PLK1 inhibitor BI 2536 may be active against CCA cells in vitro. The present study further demonstrated that PLK1 was significantly upregulated in CCA tissues and its overexpression was indicative of poor survival. Furthermore, novel drug selection based on prognosis-associated genes may provide a comprehensive insight into anti-CCA therapy. The molecules identified in the present study all displayed high binding affinity with PLK1.

However, there remain a number of limitations to the present study. First, various novel potential drugs for CCA were identified based on alterations in the genome expression landscape of patients with CCA; however, examination of the functional characterization and underlying molecular mechanisms is required in the future. Second, despite the fact that the molecular docking analysis provided binding forces between molecules and targets, the complex mechanisms and models of the specific interactions ought to be confirmed by future experiments.

In conclusion, the present study first screened the differentially expressed genes involved in the prognosis of CCA. Second, GO and KEGG gene enrichment analysis was used to determine the pathway of prognosis in CCA. A network targeted at prognostic targets was built. Finally, certain molecules were screened using the CMap database for the prognosis of CCA as a whole. These molecules may offer novel insights into the prognosis of CCA and may offer perspectives on the future innovative treatment of CCA.

\section{Acknowledgements}

The authors would like to thank the CMap, DGIdb and TCGA databases for the availability of the data.

\section{Funding}

The present study was supported by grants from the Guangxi Science and Technology Program (grant no. GuiKeAB17 195020), the Fund of National Natural Science Foundation of China (grant nos. NSFC81060202, NSFC81860319 and NSFC81260222) and Innovation Project of Guangxi Graduate Education (grant nos. YCSW2018104 and YCSW2017105).

\section{Availability of data and materials}

The datasets used and/or analyzed during the current study are available from the corresponding author on reasonable request.

\section{Authors' contributions}

The study was designed by GC, HY, YunH and PL. PL, XZZ, XDW, JJL, RQZ, YuH, YQJ and XWH were involved in the statistical analysis. PL and HY wrote the draft and GC, HY and YunH corrected the manuscript. All authors read and approved the final manuscript.

\section{Ethics approval and consent to participate}

Not applicable.

\section{Patient consent for publication}

Not applicable.

\section{Competing interests}

The authors declare that they have no competing interests.

\section{References}

1. Kaneko R, Sato Y and Kobayashi Y: Cholangiocarcinoma prognosis varies over time depending on tumor site and pathology. J Gastrointestin Liver Dis 27: 59-66, 2018.

2. Razumilava N and Gores GJ: Cholangiocarcinoma. Lancet 383 : 2168-2179, 2014.

3. Siegel RL, Miller KD and Jemal A: Cancer statistics, 2018. CA Cancer J Clin 68: 7-30, 2018.

4. Ikeno Y, Seo S, Iwaisako K, Yoh T, Nakamoto Y, Fuji H, Taura K, Okajima H, Kaido T, Sakaguchi S and Uemoto S: Preoperative metabolic tumor volume of intrahepatic cholangiocarcinoma measured by ${ }^{18} \mathrm{~F}$-FDG-PET is associated with the KRAS mutation status and prognosis. J Transl Med 16: 95, 2018.

5. DeOliveira ML, Cunningham SC, Cameron JL, Kamangar F, Winter JM, Lillemoe KD, Choti MA, Yeo CJ and Schulick RD: Cholangiocarcinoma: Thirty-one-year experience with 564 patients at a single institution. Ann Surg 245: 755-762, 2007.

6. Wang Y, Li J, Xia Y, Gong R, Wang K, Yan Z, Wan X, Liu G, Wu D, Shi L, et al: Prognostic nomogram for intrahepatic cholangiocarcinoma after partial hepatectomy. J Clin Oncol 31: 1188-1195, 2013.

7. Endo I, Gonen M, Yopp AC, Dalal KM, Zhou Q, Klimstra D, D’Angelica M, DeMatteo RP, Fong Y, Schwartz L, et al: Intrahepatic cholangiocarcinoma: Rising frequency, improved survival, and determinants of outcome after resection. Ann Surg 248: 84-96, 2008. 
8. Alvaro D, Crocetti E, Ferretti S, Bragazzi MC and Capocaccia R; AISF Cholangiocarcinoma committee: Descriptive epidemiology of cholangiocarcinoma in Italy. Dig Liver Dis 42: 490-495, 2010

9. Khan SA, Davidson BR, Goldin RD, Heaton N, Karani J, Pereira SP, Rosenberg WM, Tait P, Taylor-Robinson SD Thillainayagam AV, et al: Guidelines for the diagnosis and treatment of cholangiocarcinoma: An update. Gut 61: 1657-1669, 2012

10. Sombut S, Bunthawong R, Sirion U, Kasemsuk T, Piyachaturawat P, Suksen K, Suksamrarn A and Saeeng R: Synthesis of 14-deoxy-11,12-didehydroandrographolide analogues as potential cytotoxic agents for cholangiocarcinoma. Bioorg Med Chem Lett 27: 5139-5143, 2017.

11. Hu RW, Carey EJ, Lindor KD and Tabibian JH: Curcumin in hepatobiliary disease: Pharmacotherapeutic properties and emerging potential clinical applications. Ann Hepatol 16: 835-841, 2017.

12. Tampellini M, La Salvia A and Scagliotti GV: Novel investigational therapies for treating biliary tract carcinoma. Expert Opin Investig Drugs 25: 1423-1436, 2016.

13. Lamb J: The Connectivity Map: A new tool for biomedical research. Nat Rev Cancer 7: 54-60, 2007.

14. Wagner AH, Coffman AC, Ainscough BJ, Spies NC, Skidmore ZL, Campbell KM, Krysiak K, Pan D, McMichael JF Eldred JM, et al: DGIdb 2.0: Mining clinically relevant druggene interactions. Nucleic Acids Res 44: D1036-D1044, 2016.

15. Sangsin A, Saiudom D, Pongmanee S, Saengsin J, Leerapun T and Murakami H: Natural history and prognostic factors of cholangiocarcinoma with spinal metastasis: A 10 -year single center study. Clin Spine Surg 31: E160-E165, 2018.

16. Ouesleti S, Coutinho MF, Ribeiro I, Miled A, Mosbahi DS and Alves S: Update of the spectrum of mucopolysaccharidoses type III in Tunisia: Identification of three novel mutations and in silico structural analysis of the missense mutations. World J Pediatr 13: 374-380, 2017.

17. Pelechano V and Alepuz P: eIF5A facilitates translation termination globally and promotes the elongation of many non polyproline-specific tripeptide sequences. Nucleic Acids Res 45: 7326-7338, 2017

18. Pinho SS and Reis CA: Glycosylation in cancer: Mechanisms and clinical implications. Nat Rev Cancer 15: 540-555, 2015.

19. Jones D, Woyach JA, Zhao W, Caruthers S, Tu H, Coleman J, Byrd JC, Johnson AJ and Lozanski G: PLCG2 C2 domain mutations co-occur with $B T K$ and $P L C G 2$ resistance mutations in chronic lymphocytic leukemia undergoing ibrutinib treatment. Leukemia 31: 1645-1647, 2017.

20. Ely KA, Bischoff LA and Weiss VL: Wnt signaling in thyroid homeostasis and carcinogenesis. Genes 9: pii: E204, 2018.

21. 21 Jiang J, Protopopov A, Sun R, Lyle S and Russell M: Genomic profiling on an unselected solid tumor population reveals a highly mutated $\mathrm{Wnt} / \beta$-catenin pathway associated with oncogenic EGFR mutations. J Pers Med 8: pii: E13, 2018.

22. Mao X, Duan X and Jiang B: Fascin induces epithelial-mesenchymal transition of cholangiocarcinoma cells by regulating wnt/ $\beta$-catenin signaling. Med Sci Monit 22: 3479-3485, 2016.

23. Noll AT, Cramer T, Olde Damink SW and Schaap FG: Cholangiocarcinoma, gone without the Wnt? World J Hepatol 8: 1093-1096, 2016.

24. Steurer B, Janssens RC, Geverts B, Geijer ME, Wienholz F, Theil AF, Chang J, Dealy S, Pothof J, van Cappellen WA, et al: Live-cell analysis of endogenous GFP-RPB1 uncovers rapid turnover of initiating and promoter-paused RNA Polymerase I. Proc Natl Acad Sci USA 155: E4368-E4376, 2018.

25. Yan W, Wei J, Deng X, Shi Z, Zhu Z, Shao D, Li B, Wang S, Tong G and Ma Z: Transcriptional analysis of immune-related gene expression in p53-deficient mice with increased susceptibility to influenza A virus infection. BMC Med Genomics 8: 52 , 2015.

26. Esfandyari T, Tefferi A, Szmidt A, lain T, Zwolak P, Lasho T, Lee PW and Farassati F: Transcription factors down-stream of Ras as molecular indicators for targeting malignancies with oncolytic herpes virus. Mol Oncol 3: 464-468, 2009.

27. Munari F, Bortot A, Zanzoni S, D'Onofrio M, Fushman D and Assfalg M: Identification of primary and secondary UBA footprints on the surface of ubiquitin in cell-mimicking crowded solution. FEBS Lett 591: 979-990, 2017.

28. Lin P, Wen DY, Dang YW, He Y, Yang H and Chen G: Comprehensive and integrative analysis reveals the diagnostic, clinicopathological and prognostic significance of polo-like kinase 1 in hepatocellular carcinoma. Cell Physiol Biochem 47: 925-947, 2018.
29. Li H, Wang H, Sun Z, Guo Q, Shi H and Jia Y: The clinical and prognostic value of polo-like kinase 1 in lung squamous cell carcinoma patients: Immunohistochemical analysis. Biosci Rep: Jul 19, pii: BSR20170852, 2017 (Epub ahead of print).

30. Lin P, Xiong DD, Dang YW, Yang H, He Y, Wen DY, Qin XG and Chen G: The anticipating value of PLK1 for diagnosis, progress and prognosis and its prospective mechanism in gastric cancer: A comprehensive investigation based on high-throughput data and immunohistochemical validation. Oncotarget 8: 9249792521, 2017.

31. Gheghiani L, Loew D, Lombard B, Mansfeld J and Gavet O PLK1 activation in late G2 sets up commitment to mitosis. Cell Rep 19: 2060-2073, 2017.

32. Lin S, Hoffmann K, Gao C, Petrulionis M, Herr I and Schemmer P: Melatonin promotes sorafenib-induced apoptosis through synergistic activation of JNK/c-jun pathway in human hepatocellular carcinoma. J Pineal Res 62, 2017.

33. Dong J, Park SY, Nguyen N, Ezhilarasan R, Martinez-Ledesma E, Wu S, Henry V, Piao Y, Tiao N, Brunell D, et al: The polo-like kinase 1 inhibitor volasertib synergistically increases radiation efficacy in glioma stem cells. Oncotarget 9: 10497-10509, 2018.

34. Zheng DW, Xue YQ, Li Y, Di JM, Qiu JG, Zhang WJ, Jiang QW, Yang Y, Chen Y, Wei MN, et al: Volasertib suppresses the growth of human hepatocellular carcinoma in vitro and in vivo. Am J Cancer Res 6: 2476-2488, 2016.

35. Gopalakrishnan B, Cheney C, Mani R, Mo X, Bucci D, Walker A, Klisovic R, Bhatnagar B, Walsh K, Rueter B, et al: Polo-like kinase inhibitor volasertib marginally enhances the efficacy of the novel Fc-engineered anti-CD33 antibody BI 836858 in acute myeloid leukemia. Oncotarget 9: 9706-9713, 2018.

36. Liu X: Targeting Polo-like kinases: A promising therapeutic approach for cancer treatment. Transl Oncol 8: 185-195, 2015.

37. Van den Bossche J, Lardon F, Deschoolmeester V, De Pauw I, Vermorken JB, Specenier P, Pauwels P, Peeters M and Wouters A: Spotlight on volasertib: Preclinical and clinical evaluation of a promising Plk1 inhibitor. Med Res Rev 36: 749-786, 2016.

38. Sanaei M, Kavoosi F, Roustazadeh A and Golestan F: Effect of genistein in comparison with trichostatin a on reactivation of DNMTs genes in hepatocellular carcinoma. J Clin Transl Hepatol 6: 141-146, 2018.

39. Shang Y, Zhou Q, Wang T, Jiang Y, Zhong Y, Qian G, Zhu T, Qiu X and An J: Airborne nitro-PAHs induce Nrf2/ARE defense system against oxidative stress and promote inflammatory process by activating PI3K/Akt pathway in A549 cells. Toxicol In Vitro 44: 66-73, 2017.

40. Kikkawa Y, Takaki S, Matsuda Y, Okabe K, Taniguchi M, Oomachi K, Samejima T, Katagiri F, Hozumi K and Nomizu M: The influence of Tribenoside on expression and deposition of epidermal laminins in HaCaT cells. Biol Pharm Bull 33: 307-310, 2010.

41. Kubicsek T, Kazy Z and Czeizel AE: Teratogenic potential of tribenoside, a drug for the treatment of haemorrhoids and varicose veins-a population-based case-control study. Reprod Toxicol 31: 464-469, 2011.

42. Bhukhai K, de Dreuzy E, Giorgi M, Colomb C, Negre O, Denaro M, Gillet-Legrand B, Cheuzeville J, Paulard A, Trebeden-Negre H, et al: Ex Vivo selection of transduced hematopoietic stem cells for gene therapy of $\beta$-hemoglobinopathies. Mol Ther 26: 480-495, 2018.

43. Chang L, Hagel JM and Facchini PJ: Isolation and characterization of O-methyltransferases Involved in the biosynthesis of glaucine in glaucium flavum. Plant Physiol 169: 1127-1140, 2015.

44. Mi G, Gao Y, Yan H, Jin X, Ye E, Liu S, Gong Z, Yang H and Yang Z: 1-Scoulerine attenuates behavioural changes induced by methamphetamine in zebrafish and mice. Behav Brain Res 298: 97-104, 2016.

45. Shi J, Jiang S, Qiu D, Le W, Wang X, Lu Y and Liu Z: Rapid identification of potential drugs for diabetic nephropathy using whole-genome expression profiles of glomeruli. Biomed Res Int 2016: 1634730, 2016

46. Thrum S, Lorenz J, Mössner J and Wiedmann M: Polo-like kinase 1 inhibition as a new therapeutic modality in therapy of cholangiocarcinoma. Anticancer Res 31: 3289-3299, 2011.

This work is licensed under a Creative Commons Attribution-NonCommercial-NoDerivatives 4.0 International (CC BY-NC-ND 4.0) License. 